\title{
Cryogenic optical position encoders for mechanisms in the JWST optical telescope element simulator (OSIM)
}

\author{
Douglas B. Leviton ${ }^{\mathrm{a}^{\mathrm{*}}}$, Thomas Anderjaska ${ }^{\mathrm{b}}$, James Badger ${ }^{\mathrm{c}}$, Tom Capon ${ }^{\mathrm{a}}$, Clinton Davis ${ }^{\mathrm{d}}$, \\ Brent Dicks ${ }^{c}$, William Eichhorn ${ }^{\mathrm{a}}$, Mario Garza ${ }^{\mathrm{d}}$, Corina Guishard ${ }^{\mathrm{a}}$, Shadan Haghani ${ }^{\mathrm{b}}$, \\ Claef Hakun ${ }^{\mathrm{a}}$, Paul Haney ${ }^{\mathrm{a}}$, David Happs ${ }^{c}$, Lars Hovmand ${ }^{\mathrm{b}}$, Madhu Kadari ${ }^{\mathrm{e}}$, \\ Jeffrey Kirk ${ }^{d}$, Richard Nyquist ${ }^{c}$, F. David Robinson ${ }^{d}$, Joseph Sullivan ${ }^{c}$, Erin Wilson ${ }^{f}$
}

aNASA Goddard Space Flight Center, 8800 Greenbelt Rd., Greenbelt, MD, USA 20771

borthrop Grumann Aerospsace Systems, 4276 Forbes Blvd Lanham, MD USA 20706

${ }^{\mathrm{c}}$ Ball Aerospace and Technologies Corporation, 1600 Commerce Street Boulder, CO USA 80301

dOrbital Sciences Corporation, 7500 Greenway Center Dr., Suite 1500, Greenbelt, MD USA 20770

'Jackson and Tull, 7375 Executive Place, Suite 200, Seabrook, MD USA 20706

${ }^{\mathrm{f}}$ Genesis Engineering Solutions, 4501 Boston Way, Lanham, MD USA 20706

\begin{abstract}
The JWST Optical Telescope Element Simulator (OSIM) is a configurable, cryogenic, optical stimulus for high fidelity ground characterization and calibration of JWST's flight instruments. OSIM and its associated Beam Image Analyzer (BIA) contain several ultra-precise, cryogenic mechanisms that enable OSIM to project point sources into the instruments according to the same optical prescription as the flight telescope images stars - correct in focal surface position and chief ray angle. OSIM's and BLA's fifteen axes of mechanisms navigate according to redundant, cryogenic, absolute, optical encoders - 32 in all operating at or below $100 \mathrm{~K}$. OSIM's encoder subsystem, the engineering challenges met in its development, and the encoders' sub-micron and sub-arcsecond performance are discussed.
\end{abstract}

Keywords: cryogenic, absolute optical encoder, pattern recognition encoder, centroid, OSIM, beam image analyzer

\section{INTRODUCTION}

The James Webb Space Telescope (JWST) Optical Telescope Element Simulator (OSIM) ${ }^{1,2}$ is a massive, configurable, cryogenic, optical stimulus for JWST's flight instrument complement - called the Integrated Science Instrument Module (ISIM) - and allows high fidelity ground characterization and calibration of the instruments (Figure 1). Using a collection of remotely operated point sources, a single spherical primary mitror, and several ultra-precise, cryogenic mechanisms, OSIM projects images of those sources to ISIM as if they were stars in the sky according to the same optical prescription as the flight OTE would.

A large, X-Y-Z-tip-tilt mechanism known as the Beam Image Analyzer (BIA) carries a point-diffraction interferometer (PDI), phase retrieval camera (PHARET), and radiometer assembly - tools that allow OSIM to be configured to establish the correct optical prescription for each field point and chief ray angle and to calibrate OSIM's output flux. Figure 2 shows the BIA rail system and the tip-tilt mechanism with its angular encoders. The BIA surveys the telescope's focal surface, calibrating OSIM for all required ground observations with ISIM. OSIM itself contains two X-Y-Z stages (the Source Plate Mechanism (SPM) and Pupil Translation Mechanism (PTM)), two rotary pupil selection wheels (Pupil Select Mechanism carried by the PTM), and a two-axis gimbal for Fold Mirror 3 (Figure 3).

Each mechanism in OSIM and in the BIA navigates according to redundant, cryogenic, absolute, optical encoders -32 in all operating at temperatures at or below $100 \mathrm{~K}$. OSIM's cryogenic encoder subsystem has evolved to a large degree based on heritage from similar encoder systems for ground support equipment (GSE) for other NASA missions including the Hubble Space Telescope (HST). Those systems employed the Leviton absolute, pattern recognition encoder

doug.leviton@nasa.gov, phone 1-301-286-3670, FAX 1-301-286-0204 
technology developed at the Goddard Space Flight Center (GSFC) in the late 1990's. ${ }^{3,4}$ These encoders are unique in their method of operation in that they record and process digital images of special, absolute encoder scales to derive position in an exceptionally linear fashion with ultra-high resolution - in the few tens of nanometers in the unmagnified versions used in OSIM. ${ }^{5}$ A more thorough review of encoder operation appears in Section 3.

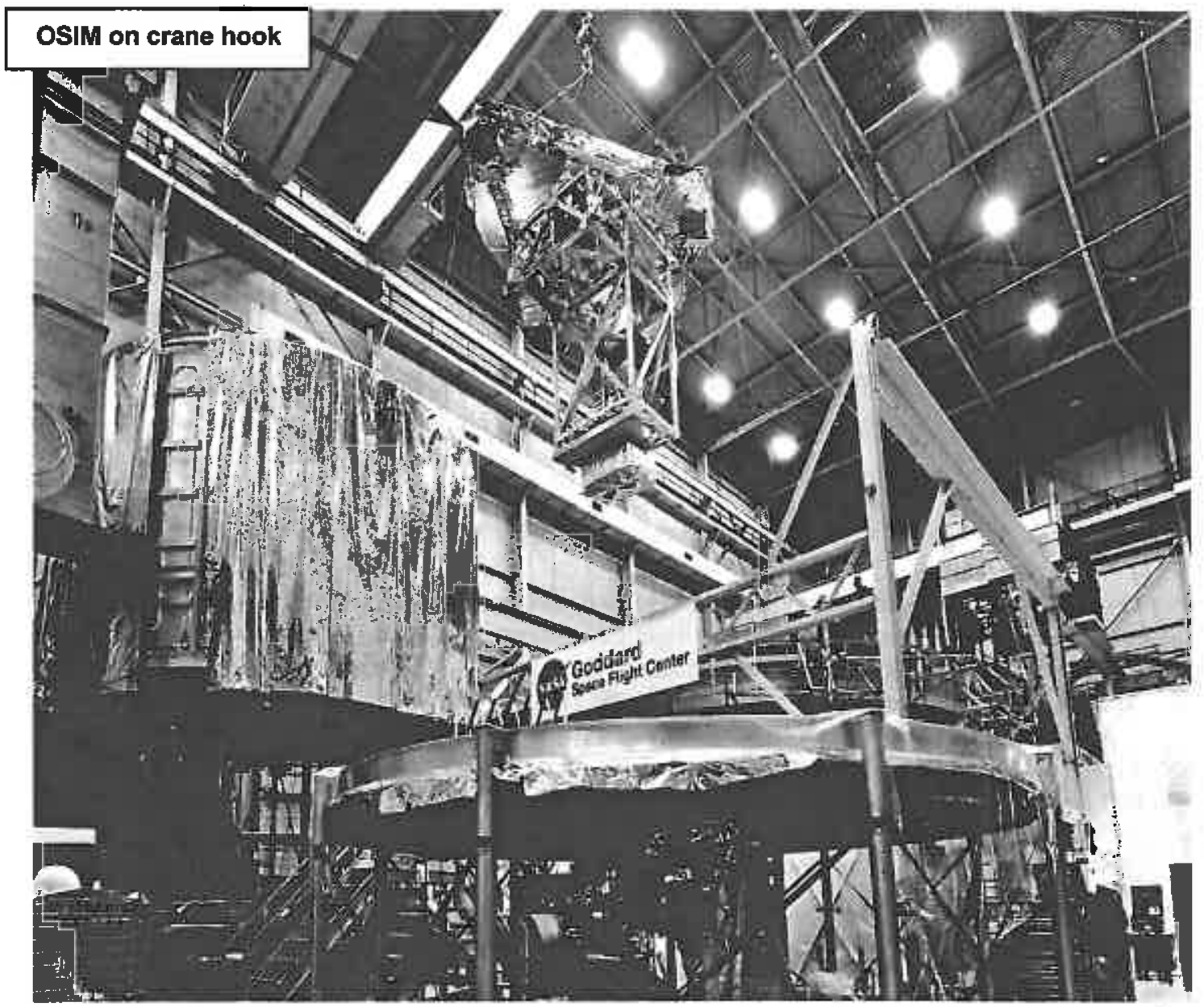

Figure 1 - OSIM is hoisted into the Space Environment Simulator (SES) cryo-vacuum chamber at NASA's Goddard Space Flight Center
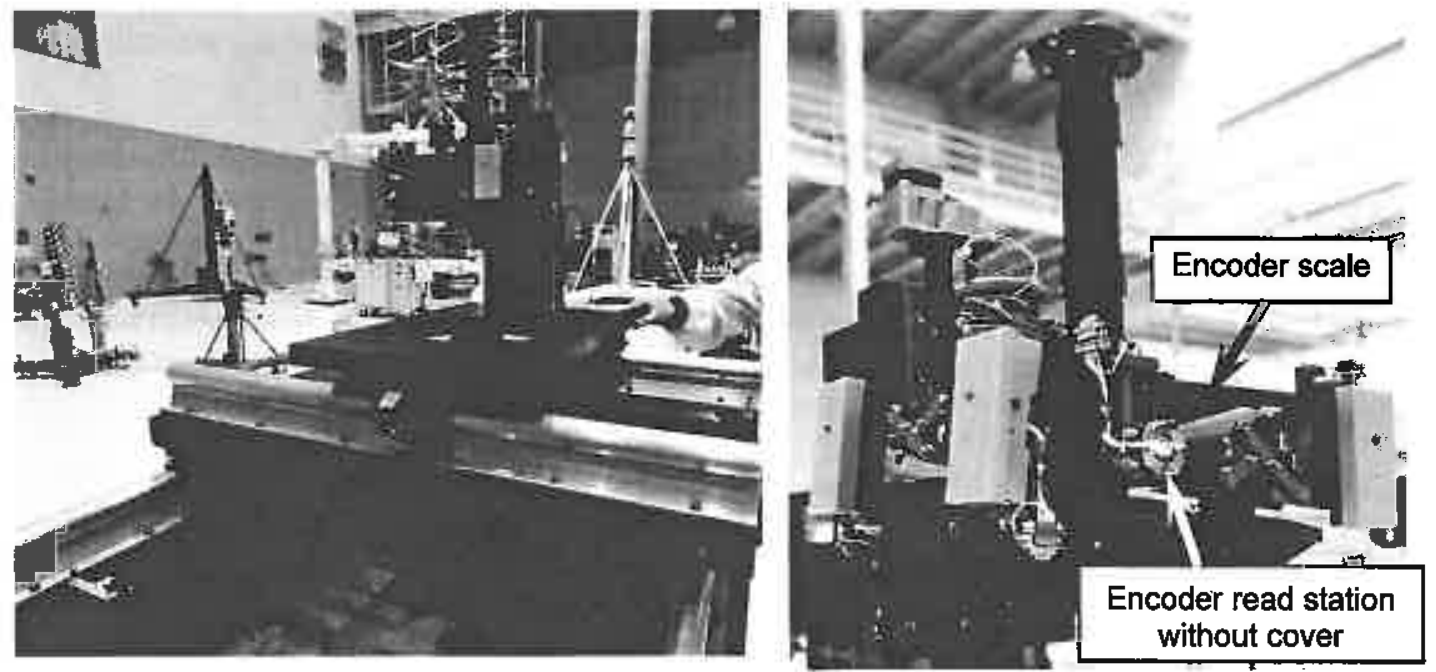

Figure 2 - left) BIA's linear X-Y-Z stage rails; right) BIA tip-tilt mechanism with runout cancelling encoders installed 

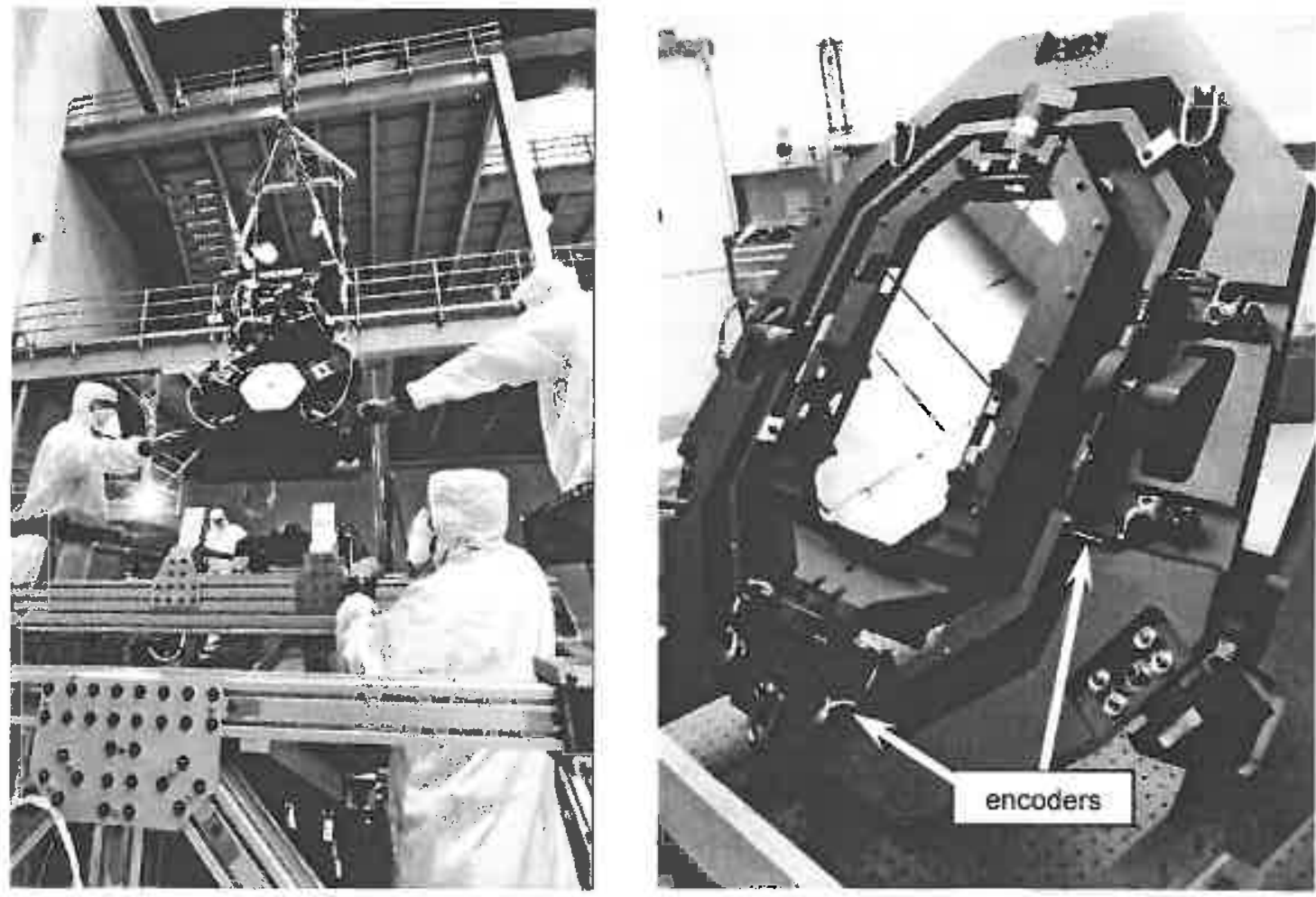

Figure 3 - left) the PTM/PSM assembly is lowered into the OSIM optical bench; right) Fold Mirror 3 in its gimbal

\section{OSIM ENCODER REQUIREMENTS}

The OSIM encoders must meet fairly challenging requirements, especially given that they must operate in a cryogenic environment. These requirements sub-allocated from system level specifications for each mechanism are tabulated in general terms and then by mechanism (Table 1).

Table 1 - OSIM encoder requirements

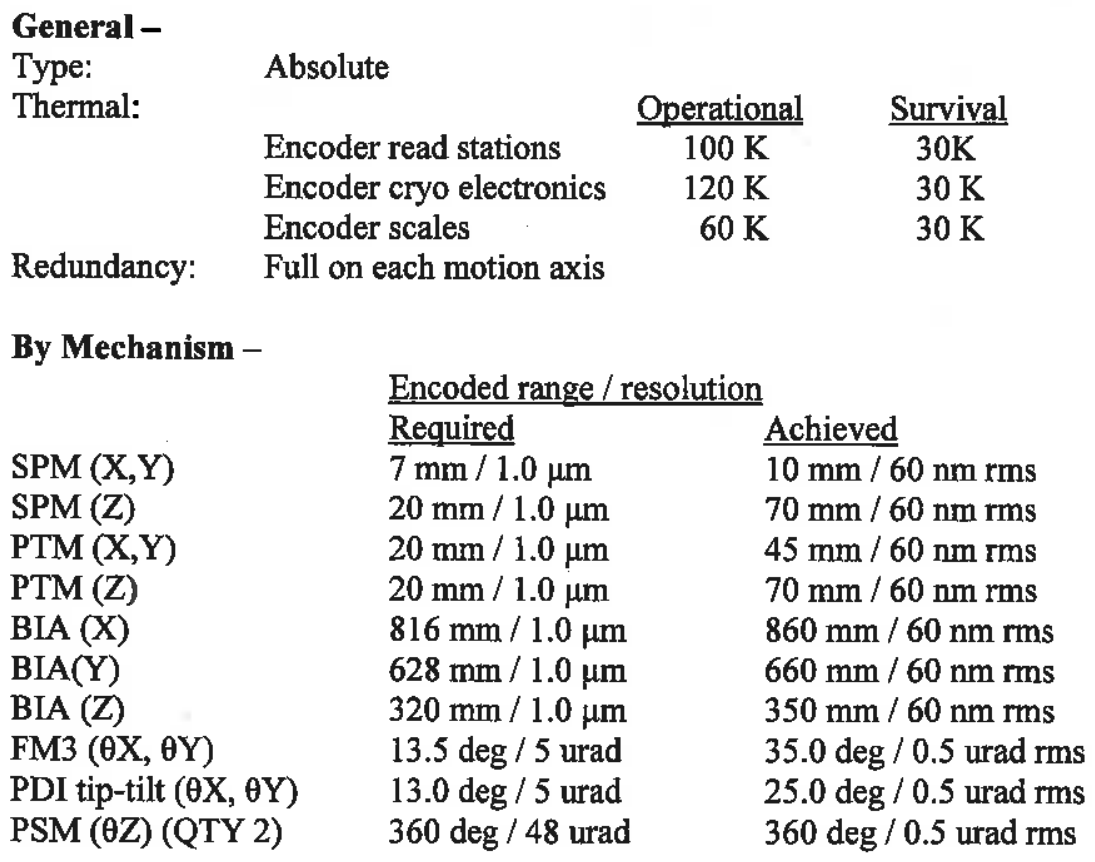




\section{ENCODER OPERATING PRINCIPLE}

Leviton absolute, pattern recognition encoders are different from conventional optical encoders in their basic method of determining position. An easy way to understand how these encoders work is to imagine a semi-infinite row of identical townhouses, stretching along US Interstate 95 from Baltimore, MD to Boston, MA as illustrated in Figure 4. Each townhouse owner is required to keep lamps lit behind its window panes that sequentially identify his street address. A passerby takes a picture of the townhouses she sees as she travels along the highway with her SmartPhone (Figure 5).

An "App" on the SmartPhone recognizes the address of each house in the picture by its lamps and measures the apparent position of its vertical rainspout with respect to the camera's pixel columns with extremely high finesse. Each house's position is computed, and the positions so computed for all houses in the picture are averaged to determine the passerby's location on the highway accurately to about $1 / 10,000^{\text {th }}$ of the width of a house (rms) or $2 / 3 \mathrm{~mm}$ ! Assuming the houses in Figure 5 are spaced by exactly $6 \mathrm{~m}$, then her location is measured as $394 \mathrm{~km}, 50 \mathrm{~m}, 378.7 \mathrm{~mm}$ from Baltimore. With each house's 24 window panes, this method encodes distances $2 \frac{1}{2}$ times around the Earth !

The linear encoder scale's pattern resembles this arrangement of identical townhouses (Figure 6). Each line in the pattern's periodic series of lines has a unique, sequential set of identifying features (binary-coded-decimal "code bits"). A camera fixed to a mechanism's base images a portion of the pattern which travels on the moving part of a mechanism (or vice versa). The camera determines the centroid of the vertically-integrated line brightness for each line with respect to its fixed pixel columns in a linear fashion and with ultra-high sensitivity which scales directly with pattern size and image magnification. A linear encoder with $10 \mathrm{X}$ magnification can have single digit nanometer resolution, while a 200 $\mathrm{mm}$ diameter rotary encoder with $10 \mathrm{X}$ magnification can have sub- 100 nrad resolution, equivalent to a 26 bit encoder in the parlance of conventional encoders.

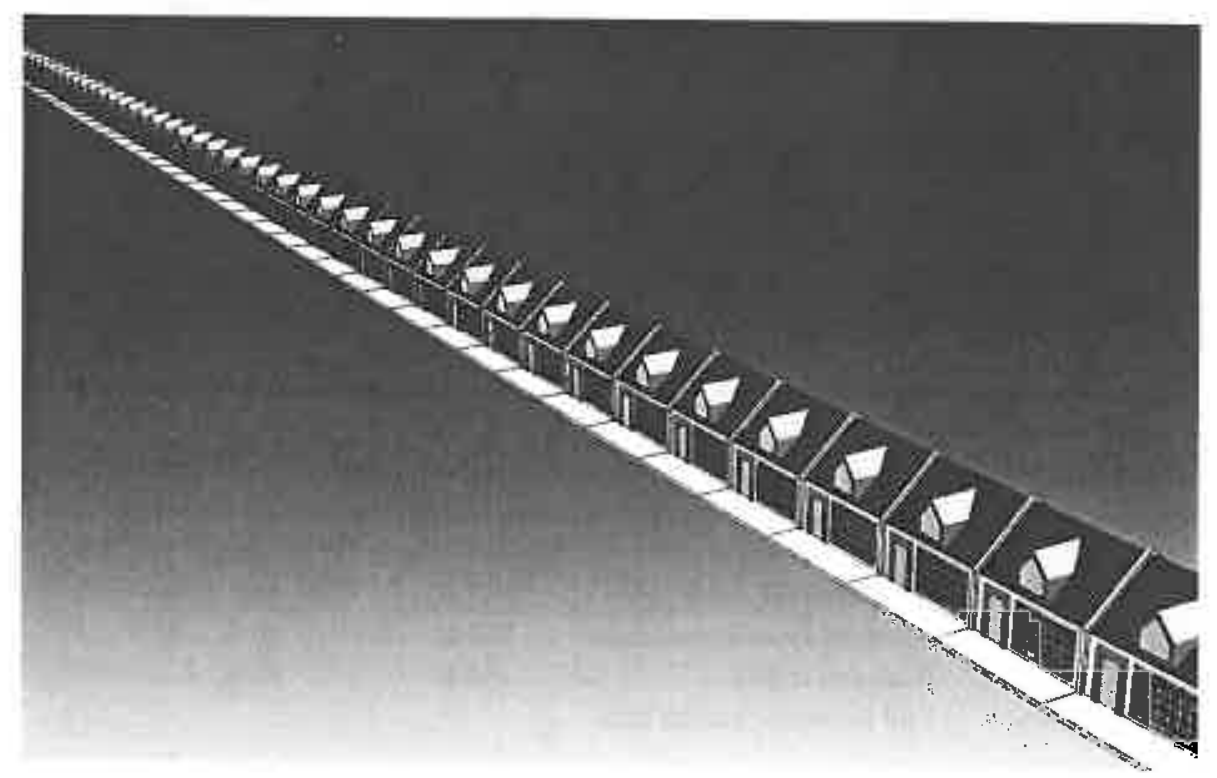

Figure $4-$ a line of identically constructed townhouses spaced by $6 \mathrm{~m}$ along a very long, straight highway

Angular encoder scales are simply fanned versions of linear patterns where the fanning is not so much that the image process would misidentify the pattern's lines. The Cartesian encoder is the two-dimensional analogue of the linear concept. In that encoder, each cell contains a lower group and an upper group of line-identifying code bits. The lower group identifies the line to left of the cell, and the upper group identifies the line at the bottom of the cell (Figure 6).

OSIM "shadow mode" encoder read stations comprise an LED light source $50 \mathrm{~mm}$ or more from an image sensor with an encoder scale interposed between the two with the scale pattern in close proximity (within $1 \mathrm{~mm}$ ) of the image sensor - the closer the better without contact. A $200 \mu \mathrm{m}$ diameter pinhole in front of the LED approximates a collimated point source. In this alignment-tolerant configuration, an adequately sharp shadow of a local portion of the scale pattern is cast on the image sensor, and the encoder image process navigates the image for specific features and forms operands which result in a position measurement. Figure 7 shows read station assemblies for several of OSIM's mechanisms. 


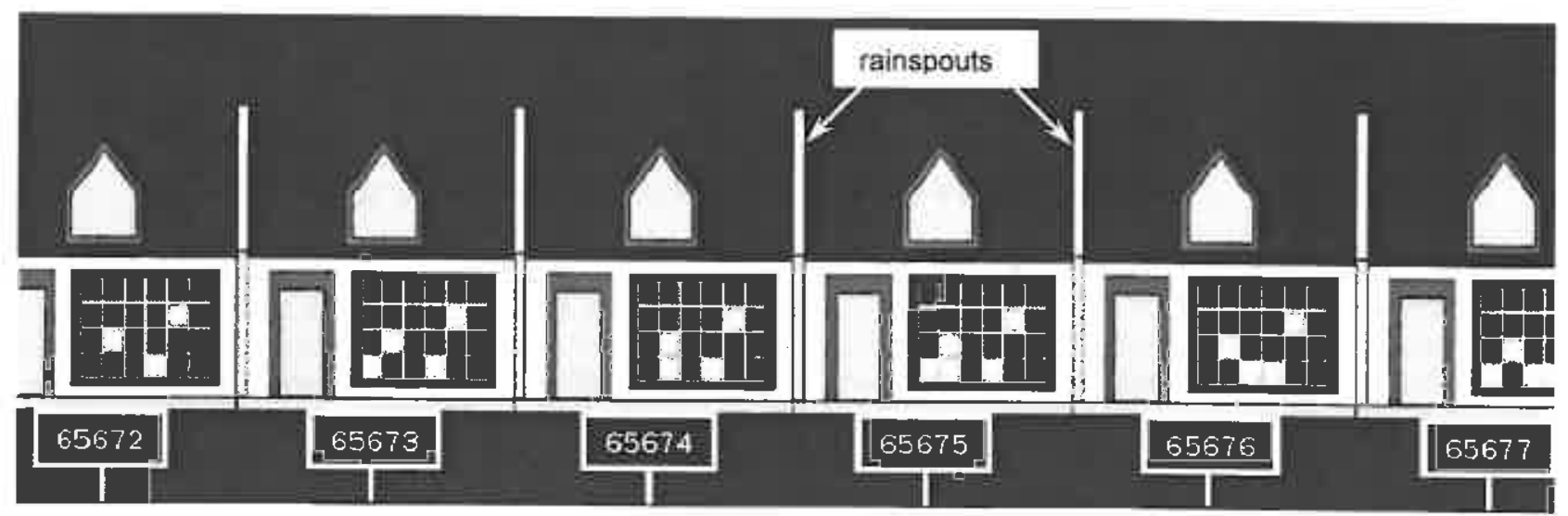

Figure 5 - digital photo of several adjacent townhouses. An image process recognizes the address of each house by its lamps and measures the apparent position of the rainspouts with respect to the camera's pixel columns with extremely high finesse:
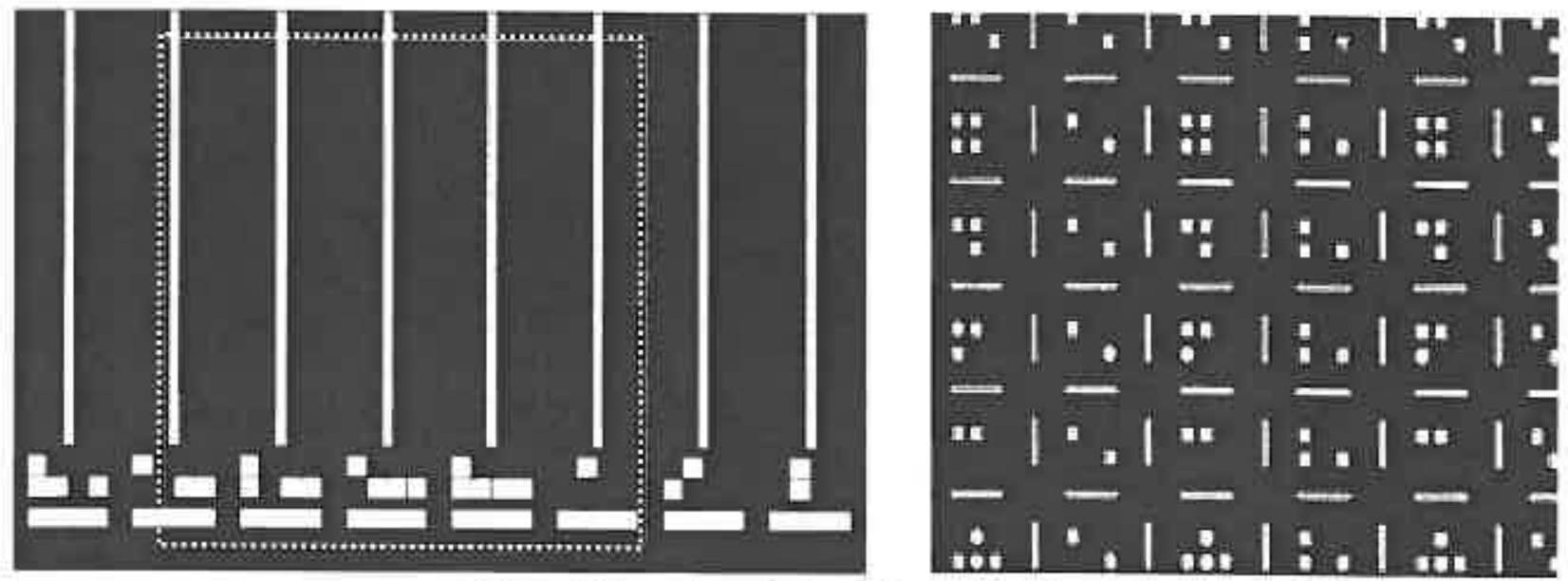

Figure 6-Leviton encoder scale patterns: left) a portion of a classic linear pattern; image process uses horizontal bars near bottom of image area (dotted square) for row navigation; right) actual Cartesian encoder scale image on a CCD imsge sensor
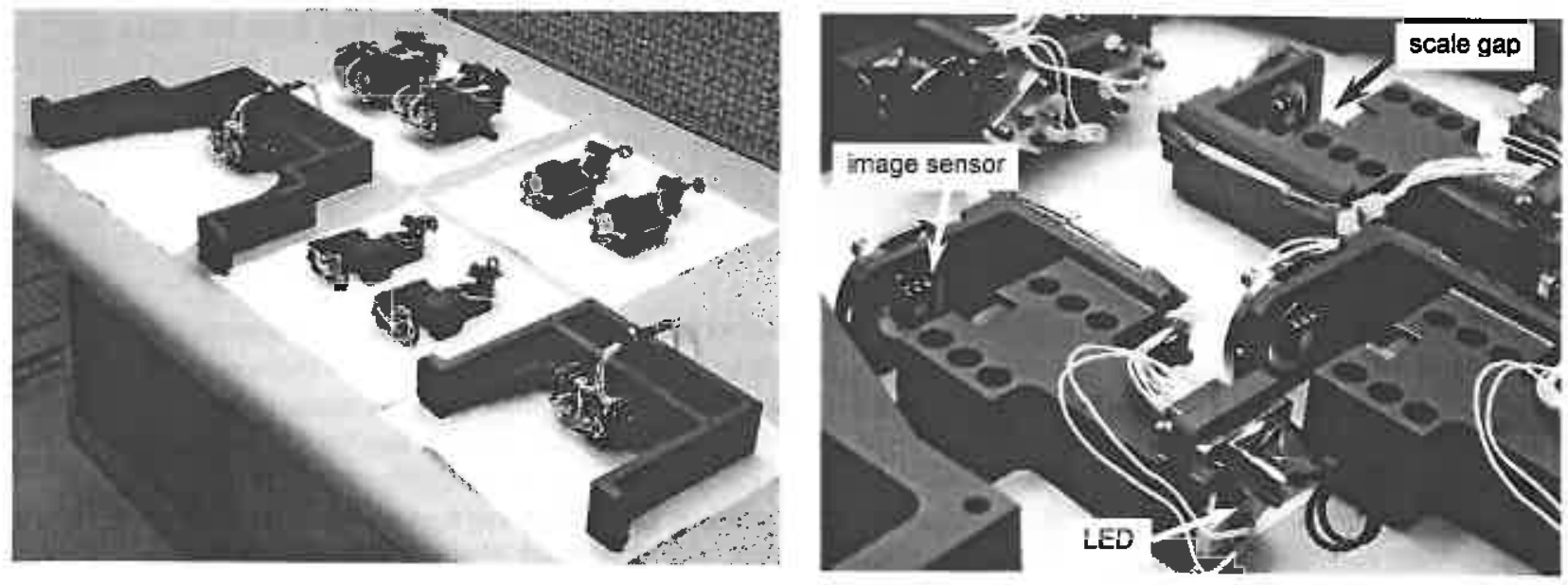

Figure 7 - left) encoder read station assemblies for SPM, PTM, and FM3; right) close-up view of FM3 read stations

On each of OSIM's angular mechanisms, a pair of diametrically opposed read stations algebraically cancels runout and provides a degree of redundancy. The mechanical axes for both the BIA tip-tilt mechanism and the Fold Mirror 3 gimbal are defined by flexural pivots which, once assembled and loaded always in the same orientation, provide very repeatable axis trajectory. Owing to this repeatability, once calibrated, a single encoder can provide accurate mechanism angle even 
if its redundant, runout-cancelling counterpart fails. For the PSM wheel assemblies, angle accuracy and runout cancellation are not so critical since the encoders are used mainly to assure that the wheels always stop at discrete, mechanical detents.

\section{EVOLUTION OF ELECTRONIC ARCHITECTURE}

The earliest Leviton encoder system for NASA GSE - the RAS/Cal Automated Multi-point Platform (RAMP) - was a large, three-axis linear translator with four absolute encoders for accurately translating the $160 \mathrm{~kg}$, reflective aberration simulator/calibrator (RAS/Cal) for HST's Advanced Camera for Surveys (ACS). RAMP operated in a cleanroom only $4.5 \mathrm{~m}$ from its encoder electronics which used commercially-available, EDC-1000 ISA-bus encoder camera cards from Electrim Corporation which connected to a camera header board with the CCD plugged into a socket on the board.

Later, an encoder system for the Calibration Stimulus from Leftover Equipment (CASTLE) was built to redundantly, absolutely encode three axes of source translation and four axes of beam steering for the calibration stimulus for HST's Wide Field Camera 3 (WFC3). CASTLE was to a significant degree a refurbishment of the calibration stimulus for HST's Wide Field and Planetary Camera 2 (WF/PC2), hence the name. The encoder system contained 11 encoders and represented the first deployment of the newly-invented, absolute, Cartesian encoder which measures two linear axes of motion in a strictly orthogonal fashion, despite arbitrary skewing of motion axes. ${ }^{6}$ CASTLE's encoders were located in a thermal vacuum chamber at room temperature about $24 \mathrm{~m}$ from the encoder readout electronics. This necessitated implementation of custom, differential, video/clock transceiver circuitry for noise immunity for the camera's video output between the Electrim ISA-bus cards and the Texas Instruments TC211 CCD image sensors used in EDC-1000 cameras. In this implementation, the CCD was outboarded from the transceiver card on a $125 \mathrm{~mm}$, six wire pigtail.

For OSIM, the requirement for the encoder image sensors to be even more remote and in a cryogenic environment necessitated further changes in electronic architecture. ${ }^{7}$ OSIM's encoder subsystem development was dramatically simplified by the early and fortunate discovery that the TC211 CCD used previously continues to produce excellent encoder scale images down to $75 \mathrm{~K}$. The TC211 is a rugged and very simple to operate CCD, having only 6 leads for two clocks, a fully buffered video output signal, power, and ground. Around the start of OSIM's development however, we found that the Electrim Corporation would soon cease to do business. This would mean an end to the ISA-bus encoder camera solution.

Meanwhile, abandoning the ISA-bus interface offered several advantages as we were free to design new flexibility into the system. First, we would be able to accommodate the much larger number of encoders required by OSIM's more elaborate set of mechanisms. We would also be able to more easily accommodate timing of analog-to-digital conversion (ADC) of pixel data in the CCD's video signal. With the image sensors now more than $30 \mathrm{~m}$ from the drive electronics, temporal latency in the video signal with respect to the local $A D C$ clock causes the ADC to miss the valid portion of the video signal. In the new architecture, the ADC clock signal is sent out and back to the image sensor so that its latency matches that of the video signal and correct timing is guaranteed. Most importantly, however, we had an opportunity to design an encoder GSE architecture that would be maintainable into the future. Finally, we might have an opportunity to take advantage of the $12 \mathrm{MHz}$ pixel clock rating of the TC211 compared to the $600 \mathrm{kHz}$ pixel clock implemented in the EDC-1000 system. This would have the potential to improve encoder data throughput.

The basic architecture of the OSIM encoder subsystem's electronics starts with a National Instruments PXI chassis that is expandable and accommodates a variety of system boards for high speed analog-to-digital conversion of encoder image sensor video and signal multiplexing as well as power distribution. This chassis communicates with a PC using the PCI bus and is programmed with a combination of LabView and $\mathrm{C}++$ for encoder function. Custom, field programmable gate array (FPGA) logic generates all clock signals for image acquisition and encoder multiplexing. Among various improvements over previous architectures made possible by these new electronics are automatic adjustments of video signal gain and offset, pixel clock speed, and ADC clock phase trimming.

Inside the cryogenic vacuum environment in close proximity to the encoder images sensor are differential encoder board's (DEB's). Each DEB handles four encoder channels to differentially receive and level shift CCD clocks, route power to each $C C D$ and scale illuminating LED, buffer each CCD's video signal, and pass back the ADC clock signal in synchrony with the video signal. CCD's are connected to DEB's with $0.5 \mathrm{~m}$, six conductor, coaxial pigtails. Mechanism encoders in OSIM naturally occur in groups of four which consequently determined the number of channels for each DEB. Figure 8 illustrates the evolution over time of the electronic architecture of Leviton GSE encoder systems. 

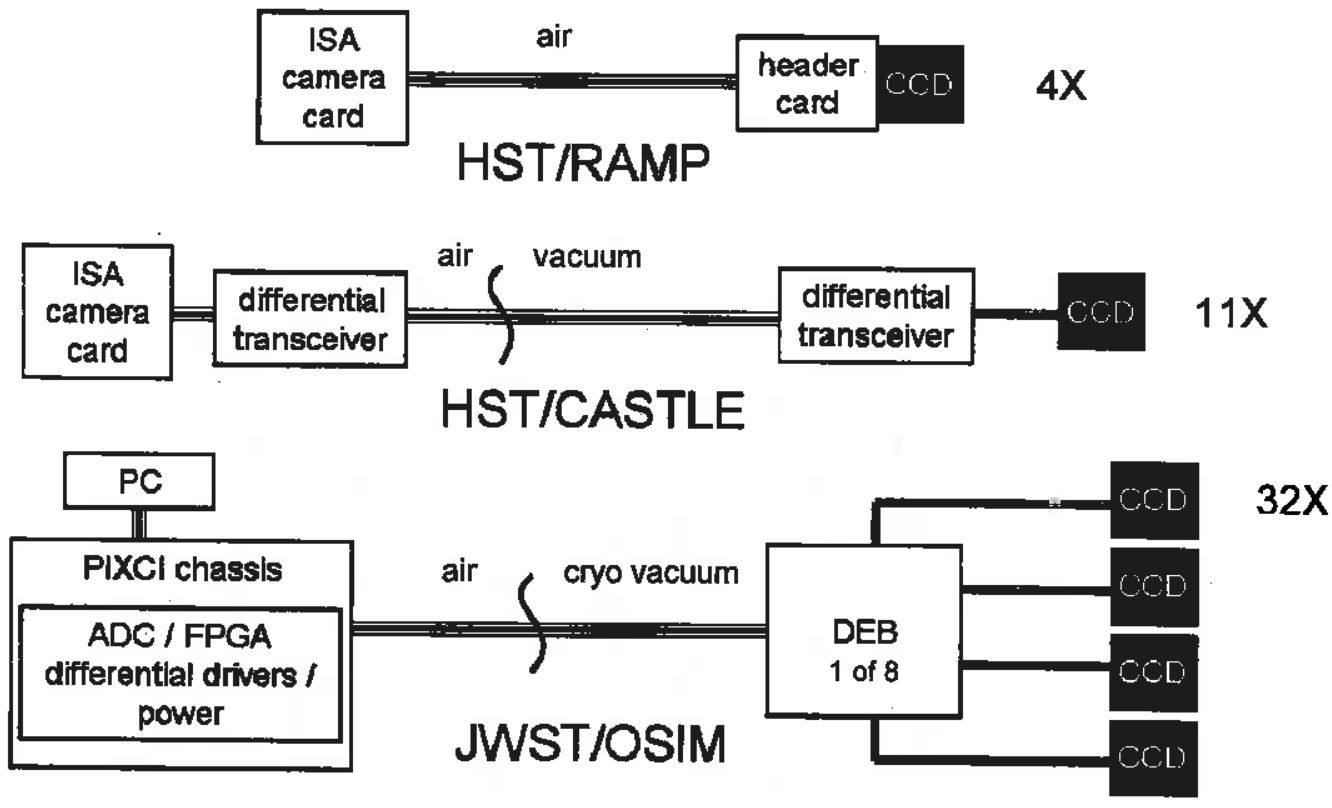

Figure 8 - evolution of Leviton encoder electronics for NASA metrology GSE; top) simplest configuration for HST/RAMP used an ISA-based clock $A D C$ card with the CCD plugged directly into a camera header card; middle) differential transceiver cards allow driving CCD clock and video signals over much longer distances up to and inside a thermal vacuum chamber for HST/CASTLE; a short pigtail eases opto-mechanical packaging of the CCD; bottom) JWST/OSIM has 32 encoder channels at greater distances yet, logically separated into eight groups of four channels; differential encoder boards (DEB's) are designed to work at $120 \mathrm{~K}$ and interface to longer CCD pigtails easing packaging and keeping thermal load of the DEB's isolated from critical optical interfaces

Each DEB contains integrated circuits and other electronic components specifically selected for reliable function at cryogenic temperatures. To assure the DEBs' reliability with OSIM in the cryogenic vacuum environment, all eight DEB's (plus spares) were cryo-cycled twice to $90 \mathrm{~K}$ and once to $30 \mathrm{~K}$ to demonstrate survival. One qualification board was cycled to $90 \mathrm{~K}$ ten times and twice to $30 \mathrm{~K}$. All TC211 CCD's installed in OSIM saw similar cycling. Several qualification CCD's were cycled many times to less than $15 \mathrm{~K}$ for survival and were demonstrated to operate down to 75 $\mathrm{K}$ where they abruptly cease to produce images. However, these CCD's would always resume producing images once warmed above $75 \mathrm{~K}$. Soda lime glass encoder scales were cycled twice to $30 \mathrm{~K}$ to demonstrate workmanship survival.

\section{ENCODER SCALE DESIGNS}

OSIM's encoder scales are made of soda lime glass. Of the photolithographic plate materials available in relatively large sizes, soda lime glass has characteristic integrated strain to cryogenic temperatures that best matches that of titanium out of which all scale holders are made. This choice of glass eases the mechanical design for encoder scale interfaces.

Each encoder scale has its line pitch and pattern features tailored specifically to the pixel grid of the TI TC211 CCD. The TC211 has 192 columns x 165 rows of rectangular pixels which are $13.75 \mu \mathrm{m}$ wide $\times 16 \mu \mathrm{m}$ high for a square image area which is $2.640 \mathrm{~mm}$ on a side. For all linear mechanisms, a pitch of $0.3900 \mathrm{~mm}$ (at $294 \mathrm{~K}$ ) was adopted. This gives 6 to 7 imaged encoder lines to generate position signal in contrast to early scale designs which gave only 3 to 4 lines. All angular scales use this same pitch with a track radius of $88.900 \mathrm{~mm}$ for an angular pitch of $\sim 4.387 \mathrm{mrad}(0.2513 \mathrm{deg})$. All Cartesian scales have a grid pitch in $X$ and $Y$ of $0.4800 \mathrm{~mm}$ versus $0.3900 \mathrm{~mm}$ allowing for more area in each grid cell to pack the additional number of code bits contained in Cartesian patterns. Cartesian scale images contain 5 or 6 lines in each direction. All imaged lines are three pixel columns wide assuring best position measuring sensitivity.

The biggest difference between classic scale design and scales designed for cryogenic application is that scales for cryo must still produce an interpretable image for the encoder image process even if mechanical shifts associated with large changes in temperature would cause the scale pattern to move vertically in the image sensor's frame. If the image would shift up too much, scale lines would shorten to the point that little or no integrated line signal would be generated for centroiding. If the pattern in the left pane of Figure 6 would move downward in the image even a little bit, the horizontal bar below each line that the image process uses for row navigation would disappear and the process would fail. 
For cryo, a continuation of each pattern line below the horizontal bars provides tolerance to vertical image shifts over temperature up to $1 \mathrm{~mm}$ (Figure 9). An encoder's image sensor is aligned so that the bars appear near the center row of the image instead of close to the bottom row like Figure 6, and the line portion below the bars generates integrated line signal for centroiding just as the original part of the line above the bars does. Now, if a downward image shift occurs, the horizontal bars remain well on the image sensor. If an upward shift occurs, signal from the lower line replaces lost signal as the upper line falls off the image sensor. The horizontal bars have also evolved into a continuous, narrow line.

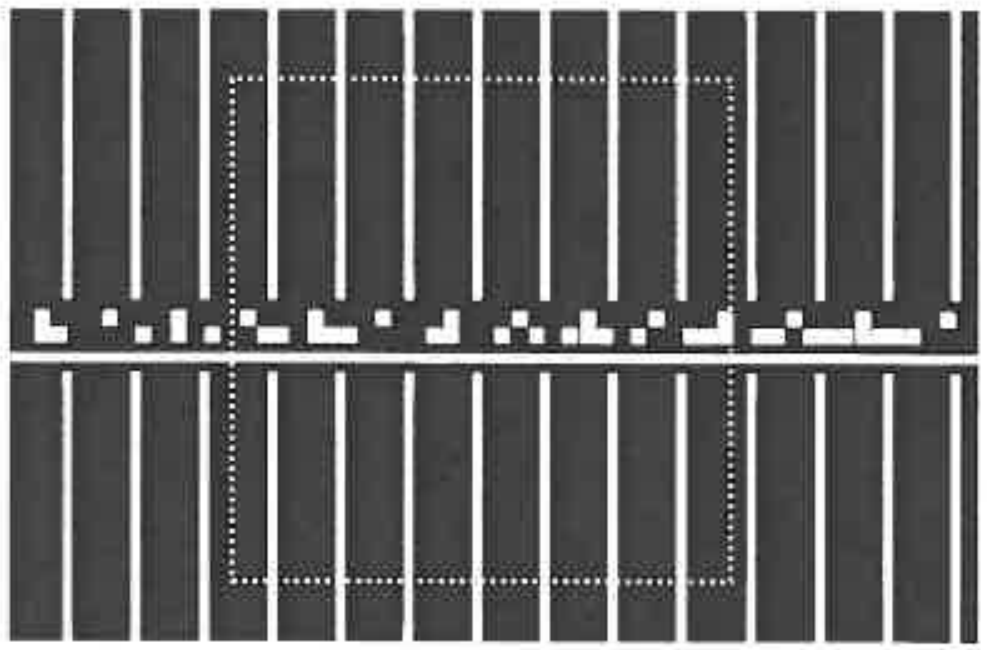

Figure 9 -revised scale pattern for cryogenic applications has continuations of pattern lines below horizontal bars (dotted square is overlay of TC211 CCD image sensor)

Each PSM wheel uses a rotary encoder disc. The BIA tip-tilt and Fold Mirror 3 gimbals use scales with pattern tracks which are sectors of that full disc. Consequently, these sector scales are rectangular in shape. The PTM uses a Cartesian scale with side-by-side patterns - one for each redundant image sensor - for its X-Y travel and a single linear scale with redundant read stations for its $Z$ travel. The SPM uses an encoder scale arrangement similar to the PTM's.

Because the widest, soda lime glass microlithography plate available is less wide at $450 \mathrm{~mm}$ than the required travel ranges for the BIA's $\mathrm{X}$ and $\mathrm{Y}$ stages, an unconventional encoding strategy is required to get past this scale length limitation. The solution found which also allows for redundancy and a smaller overall encoder package relies on pairs of reads stations disposed at each end of a stage's scale when the stage is at center of travel. At that position, all four read stations for that stage necessarily report the same position simultaneously. As the stage moves from its center of travel, continuity of that in-common position information allows the scale to travel freely - viewed by the redundant pair of read stations at one end as the pair at the other end is left behind. In rough terms, the extended travel that this arrangement provides is equal to the length of the scale plus the separation between read station pairs (Figure 10). In principle, range could be further extended by disposing additional, separated read station pairs.

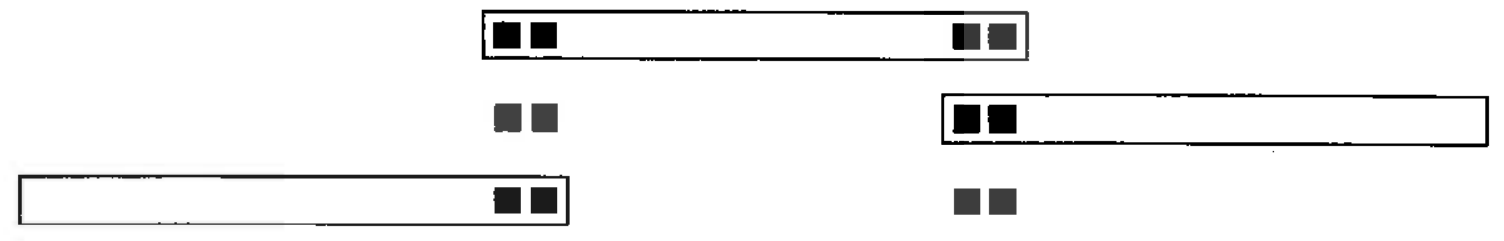

Figure $10-$ top) schematically, both redundant pairs of encoder read stations view the encoder scale with the stage at center of travel; continuity of position readout is assured as the scale ceases to be viewed by one pair of read stations or the other as the stage moves towards the positive end of travel (middle) or negative end (bottom).

\section{ENCODER PERFORMANCE}

A few typical performance aspects of OSIM's encoders and mechanisms can be seen in Figure 11. The intent of a "staircase" test at cryo is to demonstrate that a mechanism's positioning granularity requirement is met. A small part of a bi-directional scan of the PTM Z stage is shown where dozens of individual $1.5 \mu \mathrm{m}$ steps are commanded with several 
seconds passing between each step while the stage's encoder continuously records position. Not only are the $1.5 \mu \mathrm{m}$ steps accurately executed within about $20 \%$ (peak-to-peak) as measured by the encoder, but the stage relaxes immediately to its commanded position with essentially no overshoot. Noise in the encoder is at the $60 \mathrm{~nm}$ rms level.

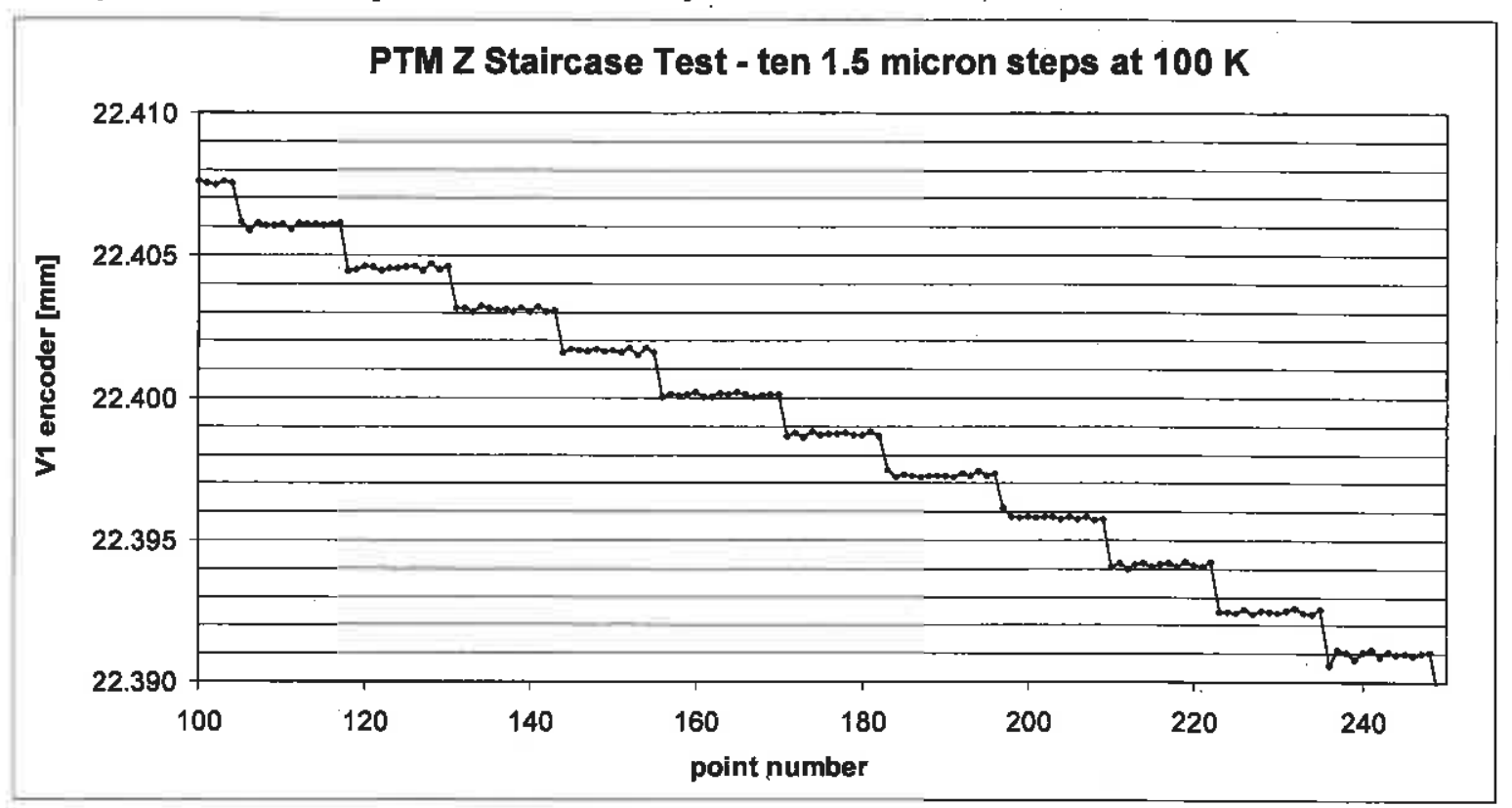

Figure 11 - a portion of encoder data stream for a staircase test of the PTM Z axis stage at cryo

Figure 12 illustrates very repeatable stage error in the PTM Y stage. During buildup of the PTM - before encoder readout offsets were critically recorded to make encoders read zero at center of travel - four $10 \mathrm{~mm}$ scans with the PTM $Y$ stæge were commanded in $0.25 \mathrm{~mm}$ increments from $-4 \mathrm{~mm}$ (on the encoder) to $+6 \mathrm{~mm}$. Stage error, arbitrarily defined to be zero at the beginning of each scan, is taken to be the difference between the encoder reading at each stop and commanded position. One can easily see that the error signature of the stage's $2 \mathrm{~mm}$ pitch lead screw is about 12 $\mu$ m peak-to-peak (6 ppt) with each revolution, but repeatable at each position to within about $1 \mu \mathrm{m}$. Small but noticeable and repeatable structures in the stage trajectory can be seen, for example, at $-1 \mathrm{~mm}, 0.25 \mathrm{~mm}$, and $+2.25 \mathrm{~mm}$. Figure 12 well demonstrates the value of the encoder to provide absolute knowledge of stage position to the required $1 \mu \mathrm{m}$ level.

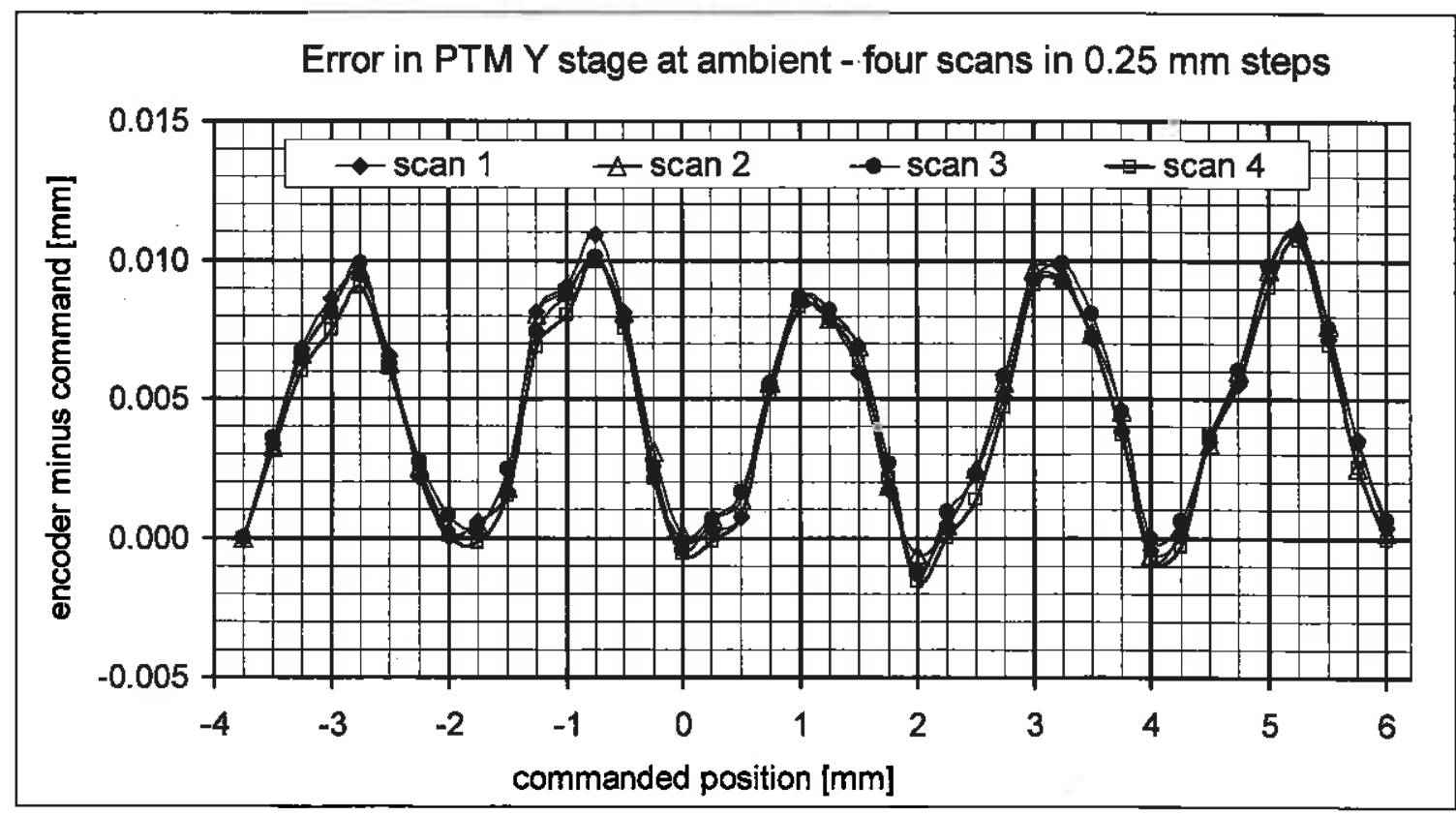

Figure 12 - error in PTM Y stage captured in four $10 \mathrm{~mm}$ scans in $0.25 \mathrm{~mm}$ increments reveal errors in $2 \mathrm{~mm}$ pitch lead screw 
An important aspect of encoder performance for accurate position measurement at cryogenic temperatures or indeed at any temperature is knowledge of the encoder scale's pitch as a function of temperature. This is most critical for the linear encoders on the BIA's very long travel stages since the BIA is the primary tool used to calibrate OSIM itself which is later used to stimulate the flight JWST instruments in a known way. A strategy was adopted to use the long encoder scales themselves along with their widely separated read stations to calibrate scale pitch with temperature.

A temperature sensor is attached to each BIA linear glass encoder scale and to aluminum structure on each stage some distance from an aluminum bracket to which each stage's read stations are mutually attached. As the BIA stages cool toward liquid nitrogen temperature, position readings are taken from all four read stations on each stage, positioned near its center of travel. Changes in the apparent separations or longest baselines between the read stations are tracked with changing scale temperature and aluminum temperature. Part of each apparent change is due to contraction in allaluminum structure separating the read stations. That component is easily accounted for using accurate, well-known thermal integrated strain data for $\mathrm{Al} \mathrm{6061.} \mathrm{Simple} \mathrm{algebra} \mathrm{reveals} \mathrm{the} \mathrm{balance} \mathrm{of} \mathrm{the} \mathrm{change} \mathrm{which} \mathrm{is} \mathrm{due} \mathrm{to} \mathrm{integrated}$ strain in the soda lime encoder glass. A new scale pitch as a function of temperature is derived from the latter, measured, integrated strain. Figure 13 compares measured integrated strain in soda lime glass with values found in the literature. ${ }^{8.9}$

Agreement amongst the data for the different stages' glass and that from the literature is remarkable considering the number of years that have passed since the published measurements were made and the relative indirectness of the present method. For the BIA measurements taken alone, the differences in derived integrated strain at any temperature from scale to scale for different stages could result from a few factors. In order of decreasing likelihood, these are: 1) thermal gradients in the aluminum structure between where each stage's temperature sensor is mounted and the mechanical connection to the aluminum bracket separating that stage's read stations; 2) inconsistency in the quality of the thermal connection between each glass scale and its temperature sensor, 3) thermal gradients in each glass scale, and 4) lack of thermal equilibrium in the BIA. These potential causes for scatter in the current data could be at play when one considers that the best agreement with literature values is for two tests of the $Z$ stage by itself (dashed curves) conducted in a simpler, more homogeneous, cryo vacuum environment in a smaller chamber than in OSIM cryo-vac testing.

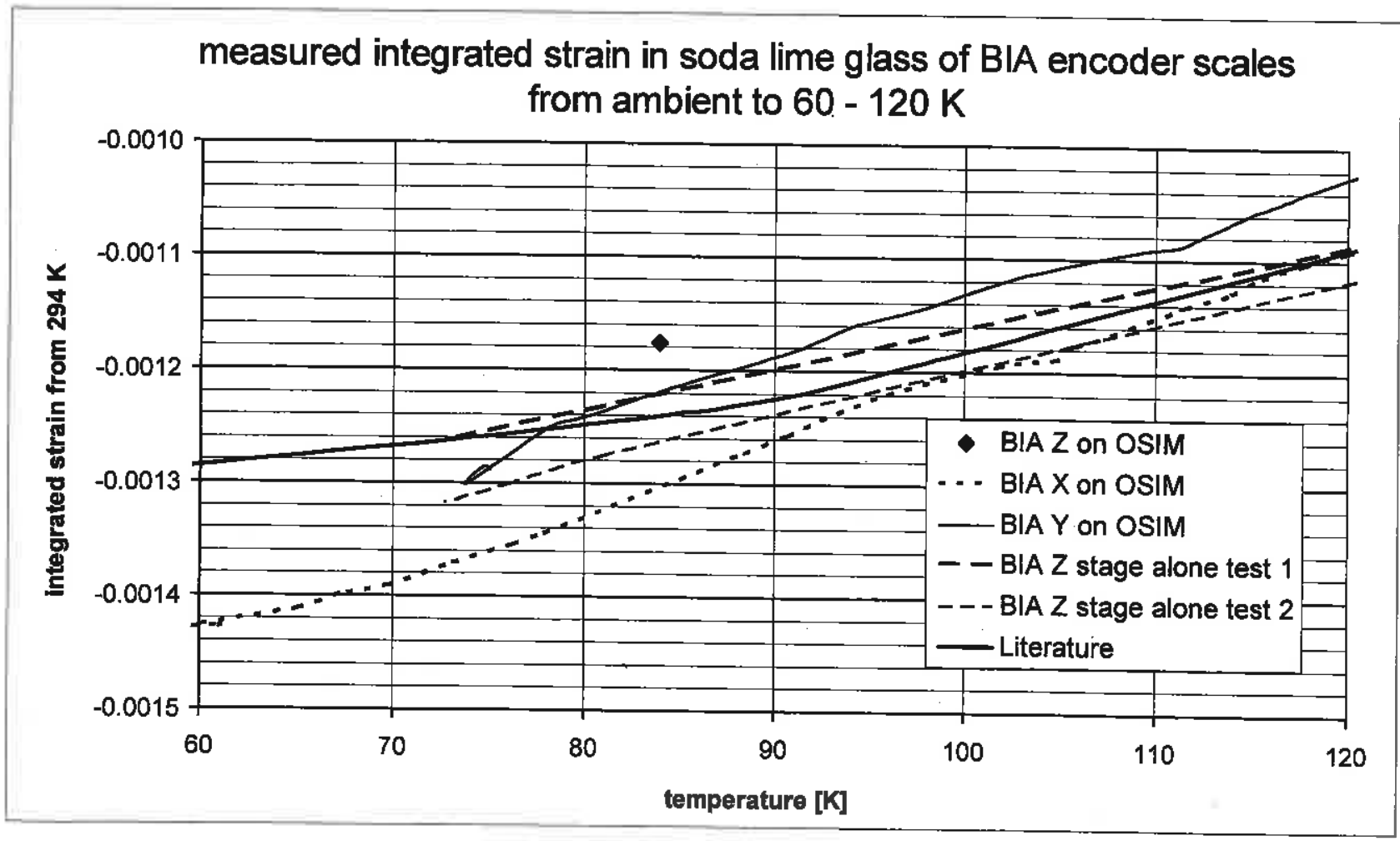

Figure 13 - comparison of measured integrated strain in soda lime glass to cryo from ambient with literature values 
For purposes of OSIM BIA operations at cryogenic temperatures, encoder scale pitch can be represented by a linear fit of pitch to glass temperature from $60-90 \mathrm{~K}$ :

$$
\text { pitch }[\mathrm{mm}]=2.96181 \mathrm{E}-06[\mathrm{~mm} / \mathrm{K}] \times \mathrm{T}[\mathrm{K}]+0.389265[\mathrm{~mm}]
$$

With this fit, we see that scale pitch changes at a rate of roughly $3 \mathrm{~nm} / \mathrm{K}$. At $75 \mathrm{~K}$, the scale is about $99.87 \%$ of its sizes at room temperature. From $60-90 \mathrm{~K}$, the peak-to-peak difference in measured encoder scale pitch amongst the three stages is $40 \mathrm{~nm}$. For simplicity, if one were to assume a single value of scale pitch for the middle of the BIA's cryogenic operational temperature range for all three axes, a peak error from the center of travel in the longest stage to either end of travel would result in an error of less than or equal to $34 \mu \mathrm{m}$.

\section{FUTURE USE OF OSIM ENCODERS}

A second BIA - known creatively as BIA2 - customized for testing the flight JWST telescope in a mammoth cryovacuum chamber at Johnson Space Center in Houston, TX is currently being built. Its encoder electronics including image sensors will be built-to-print. Although, at the time OSIM development commenced, the TI TC211 CCD was still commercially available, that has ceased to be the case. Meanwhile, we maintain enough spare CCD's from the first BIA build to complete a second unit. Texas Instruments still manufactures another relatively small format CCD in the same technology family which likely also functions at $80 \mathrm{~K}$ which could conceivably be used in place of the TC211 in future builds at the expense of significant additional non-recurring engineering to change the electronics to drive the more complex device and qualification testing for those electronics and the CCD at cryogenic temperatures.

\section{ACKNOWLEDGMENTS}

We wish to thank OSIM instrument mangers Thai Pham at GSFC, Robert Rashford of Genesis Engineering Solutions, and Paul Volmer of Ball Aerospace for their leadership in the smooth development of the encoder subsystem for OSIM.

\section{REFERENCES}

[1] Davila, P.S., et al, "The Optical Telescope Element Simulator for the James Webb Space Telescope," SPIE 7010, (2008), ISBN: 9780819472205 , http://spie.org/x648.html?product id $=790402$

[2] Sullivan, J., et al, "Manufacturing and integration status of the JWST OSIM optical simulator," SPIE 7731-139, (2010), http://spie.org/ $\times 648 . \mathrm{html}$ ?product id $=856758$

[3] Leviton, D.B., Garza, M.S., "Recent advances and applications for NASA's new, ultra-high sensitivity, absolute, optical pattern recognition encoders," SPIE 4091, 375-384, (2000), http://spie.org/x648.html?product id=405797

[4] Leviton, D.B., et al, "Wide Field Imaging Interferometry Testbed 3 - metrology subsystem ," SPIE 4852, 827-838, (2003), http://spie.org/x648.html?product id $=460948$

[5] Leviton, D.B., "Image processing for new optical pattern recognition encoders," SPIE 4113, 32-40, (2000), http://spie. 0 rg $/$ x648.html?product id $=405860$

[6] Leviton, D.B., Kirk, J., Lobsinger, L., "Ultra-high resolution Cartesian absolute optical encoder," SPIE 5190, 111$121,(2003)$, http://spie.org/x648.html?product id $=518376$

[7] Leviton, D.B., Frey, B.J., "Ultra-high resolution, absolute position sensors for cryostatic applications," SPIE 4850, 776-787, (2003), http://spie.org/x648,html?product id=461799

[8] Feltham, S.J., Yates, B., Martin, R.J., "The thermal expansion of particulate-reinforced composites," J. Materials

Science, 17, p2309 (1982), http://link.springer.com/article/10.1007/BF00543740

[9] White, G.K., "Thermal Expansion of Silica at Low Temperatures," Cryogenics, 4, pp. 2-7, (1964) 